\title{
First Scattered-light Images of the Gas-rich Debris Disk around 49 Ceti
}

\author{
Élodie Choquet ${ }^{1,19}$, Julien Milli ${ }^{2}$, Zahed Wahhaj ${ }^{2}$, Rémi Soummer ${ }^{3}$, Aki Roberge ${ }^{4}$, Jean-Charles Augereau ${ }^{5}$, Mark Booth ${ }^{6,7}$,
} Olivier Absil ${ }^{8,20}$, Anthony Boccaletti ${ }^{9}$, Christine H. Chen ${ }^{3}$, John H. Debes ${ }^{3}$, Carlos del Burgo ${ }^{10}$, William R. F. Dent ${ }^{11}$, Steve Ertel ${ }^{12}$, Julien H. Girard ${ }^{2}$, Elena Gofas-Salas ${ }^{13}$, David A. Golimowski ${ }^{3}$, Carlos A. Gómez González ${ }^{8}$, J. Brendan Hagan ${ }^{3}$, Pascale Hibon $^{2}$, Dean C. Hines ${ }^{3}$, Grant M. Kennedy ${ }^{14}$, Anne-Marie Lagrange ${ }^{5}$, Luca Matrà ${ }^{14}$, Dimitri Mawet ${ }^{1,15}$, David Mouillet ${ }^{5}$, Mamadou N'Diaye ${ }^{3,16}$, Marshall D. Perrin ${ }^{3}$, Christophe Pinte ${ }^{5}$, Laurent Pueyo ${ }^{3}$, Abhijith Rajan ${ }^{17}$, Glenn Schneider ${ }^{12}$, Schuyler Wolff ${ }^{18}$, and Mark Wyatt ${ }^{14}$

${ }^{1}$ Jet Propulsion Laboratory, California Institute of Technology, 4800 Oak Grove Drive, Pasadena, CA 91109, USA; echoquet@jpl.nasa.gov

${ }^{2}$ European Southern Observatory, Alonso de Còrdova 3107, Vitacura, Casilla 19001, Santiago, Chile

${ }^{3}$ Space Telescope Science Institute, 3700 San Martin Drive, Baltimore, MD 21218, USA

${ }^{4}$ Exoplanets \& Stellar Astrophysics Laboratory, NASA Goddard Space Flight Center, Code 667, Greenbelt, MD 20771, USA ${ }^{5}$ Univ. Grenoble Alpes, CNRS, IPAG, F-38000 Grenoble, France

${ }^{6}$ Astrophysikalisches Institut und Universitätssternwarte, Friedrich-Schiller-Universität Jena, Schillergäßchen 2-3, D-07745 Jena, Germany ${ }^{7}$ Instituto de Astrofísica, Pontificia Universidad Católica de Chile, Vicuña Mackenna 4860, Santiago, Chile

${ }^{8}$ Space sciences, Technologies and Astrophysics Research (STAR) Institute, Université de Liège, 19 Allée du Six Août, B-4000 Liège, Belgium ${ }^{9}$ LESIA, Observatoire de Paris, PSL Research University, CNRS, Sorbonne Universités, UPMC Univ. Paris 06, Univ. Paris Diderot, Sorbonne Paris Cité, 5 place Jules Janssen, F-92195 Meudon, France

${ }^{10}$ Instituto Nacional de Astrofísica, Óptica y Electrónica, Luis Enrique Erro 1, Sta. Ma. Tonantzintla, Puebla, Mexico

${ }^{11}$ Atacama Large Millimeter/submillimeter Array (ALMA) Santiago Central Offices, Alonso de Còrdova 3107, Vitacura, Casilla 763 0355, Santiago, Chile

${ }^{12}$ Steward Observatory, Department of Astronomy, University of Arizona, 933 N. Cherry Avenue, Tucson, AZ 85721, USA

${ }^{13}$ ONERA, 29 Avenue de la Division Leclerc, F-92320 Chatillon, Paris, France

${ }^{14}$ Institute of Astronomy, University of Cambridge, Madingley Road, Cambridge CB3 0HA, UK

${ }^{15}$ Department of Astronomy, California Institute of Technology, 1200 E. California Boulevard, MC 249-17, Pasadena, CA 91125, USA

${ }^{16}$ Laboratoire Lagrange, Université Côte d'Azur, Observatoire de la Côte d'Azur, CNRS, Parc Valrose, Bât. H. Fizeau, F-06108 Nice, France ${ }_{17}$ Arizona State University, Phoenix, AZ 85004, USA

18 Johns Hopkins University, 3400 North Charles Street, Baltimore, MD 21218, USA

Received 2016 November 9; revised 2016 December 19; accepted 2016 December 19; published 2017 January 9

\begin{abstract}
We present the first scattered-light images of the debris disk around 49 Ceti, a $~ 40$ Myr A1 main-sequence star at $59 \mathrm{pc}$, famous for hosting two massive dust belts as well as large quantities of atomic and molecular gas. The outer disk is revealed in reprocessed archival Hubble Space Telescope NICMOS-F110W images, as well as new coronagraphic H-band images from the Very Large Telescope SPHERE instrument. The disk extends from 1". 1 (65 au) to 4".6 (250 au) and is seen at an inclination of $73^{\circ}$, which refines previous measurements at lower angular resolution. We also report no companion detection larger than $3 M_{\text {Jup }}$ at projected separations beyond 20 au from the star (0"'34). Comparison between the F110W and H-band images is consistent with a gray color of 49 Ceti's dust, indicating grains larger than $\gtrsim 2 \mu \mathrm{m}$. Our photometric measurements indicate a scattering efficiency/infrared excess ratio of 0.2-0.4, relatively low compared to other characterized debris disks. We find that 49 Ceti presents morphological and scattering properties very similar to the gas-rich HD 131835 system. From our constraint on the disk inclination we find that the atomic gas previously detected in absorption must extend to the inner disk, and that the latter must be depleted of $\mathrm{CO}$ gas. Building on previous studies, we propose a schematic view of the system describing the dust and gas structure around 49 Ceti and hypothetical scenarios for the gas nature and origin.
\end{abstract}

Key words: circumstellar matter - stars: individual (49 Ceti) - techniques: image processing

\section{Introduction}

During their protoplanetary phase, young circumstellar environments are composed of an optically thick disk of primordial gas and dust from an original molecular cloud. Molecular gas then dominates the system over dust. Within $10 \mathrm{Myr}$ though, the gas-to-dust ratio inverts as most of the gas dissipates under the successive effects of viscous accretion onto the star, photoevaporation by stellar and interstellar radiation, and accretion by giant planets. The dust grains coagulate, grow from submicron to millimeter size, and settle in the mid-plane. Then, the disk becomes optically thin (Williams \& Cieza 2011). By then the system has turned into a gas-poor disk of debris, with large planetesimals on colliding orbits, producing second-

\footnotetext{
${ }^{19}$ Hubble Fellow.

20 F.R.S-FNRS Research Associate.
}

generation dust particles through a destructive grinding process (Wyatt 2008).

The optically thin environment in debris disks is supposed to be hostile to the persistence of primordial molecular gas, which is photodissociated on very short timescales by UV radiation. Yet, a handful of young systems exhibit a substantial amount of $\mathrm{CO}$ gas while having ages and dust properties of debris disks (Zuckerman et al. 1995; Moór et al. 2011, 2015a; Dent et al. 2014; Greaves et al. 2016; Lieman-Sifry et al. 2016; Marino et al. 2016). They all harbor large quantities of dust, indicated by their fractional infrared luminosity $L_{\mathrm{IR}} / L_{\star} \sim 1 \times 10^{-3}$ and have ages between 15 and $50 \mathrm{Myr}$ (Greaves et al. 2016). The nature and origin of their molecular gas are not completely clear: it may be primordial gas preserved from photodissociation by self-shielding, or second-generation gas released by colliding comets or planetesimals. These are fundamental 
questions to investigate, as gas plays a major role in the planet formation process.

49 Ceti is an A1V star at $59 \pm 1$ pc (van Leeuwen 2007) associated with the $\sim 40$ Myr Argus association (Zuckerman \& Song 2012). This gas-rich system, long known for its large infrared excess (Sadakane \& Nishida 1986; Jura et al. 1998), is now well characterized by two dust populations (Roberge et al. 2013, hereafter R13; Chen et al. 2014; Kennedy \& Wyatt 2014; Moór et al. 2015b): a warm inner disk $\left(\sim 155 \mathrm{~K}, L_{\mathrm{IR}} /\right.$ $\left.L_{\star} \sim 2 \times 10^{-4}\right)$ and a cold outer disk $\left(\sim 60 \mathrm{~K}, L_{\mathrm{IR}} /\right.$ $\left.L_{\star} \sim 9 \times 10^{-4}\right)$. The thermal emission of both dust belts has been resolved, at 12.5 and $17.9 \mu \mathrm{m}$ for the inner disk (Wahhaj et al. 2007) and at 70, 100, 160, and $450 \mu \mathrm{m}$ for the outer disk (R13; Moór et al. 2015b; Greaves et al. 2016). Dust is detected up to $60 \mathrm{au}$ in the inner disk and likely depleted below $30 \mathrm{au}$. The outer disk is detected up to 400 au with poor constraints on its inner radius due to the poor angular resolution achieved in the far-infrared. All images show substantially inclined disks, albeit with poor constraints on the inclination $\left(45^{\circ}-85^{\circ}\right)$.

Both molecular and atomic gas were detected in the system. $\mathrm{CO}$ emission was resolved in the outer disk at similar radial scales as the dust and appears edge-on (Hughes et al. 2008). It was not detected in the inner disk, where photochemistry models confirm that molecular gas should be photodissociated. Despite the substantial quantity of $\mathrm{CO}\left(\gtrsim 2.2 \times 10^{-4} M_{\oplus}\right)$, no CO absorption was detected in the UV (Roberge et al. 2014), indicating that the molecular disk might not be quite edge-on. Atomic gas was also detected, in the form of $\mathrm{C} \mathrm{II} \mathrm{emission,} \mathrm{as}$ well as many absorption lines (Ca II, O I, C I, C II, C IV, Fe II), some of which show significant variability, indicative of infalling star-grazing comets (Montgomery \& Welsh 2012; Roberge et al. 2013, 2014; Malamut et al. 2014; Miles et al. 2016).

Analyzing the dust composition may cast a new light on the processes at work in 49 Ceti. As suggested by R13, the high $\mathrm{C} / \mathrm{O}$ ratio might be explained by photodesorption or graingrain collision of carbon-rich dust. Although scattered-light images could help analyze its dust properties, 49 Ceti's disk has so far eluded near-infrared imagers, hinting at dust grains with very low scattering efficiency.

We present the first scattered-light images of 49 Ceti's outer disk. The disk was imaged by reprocessing archival Hubble Space Telescope (HST) NICMOS data in the F110W filter, then with the Very Large Telescope (VLT) SPHERE instrument in $\mathrm{H}$ band. The images provide a first $J-H$ color measurement for 49 Ceti dust grains. This detection adds to the nine debris disks previously reported from the ALICE program (Soummer et al. 2014; Choquet et al. 2016) and to the detection of the debris disk around HD 114082 from the SPHERE High Angular Resolution Debris Disk Survey (SHARDDS) program (Wahhaj et al. 2016). We also report exoplanet detection limits obtained with SPHERE.

\section{Observations}

The HST image comes from an archival NICMOS data set obtained on UT-2004-12-30 as part of a survey looking for circumstellar disks (HST-GO-10177; PI: G. Schneider). Owing to a previous non-detection (Weinberger et al. 1999), 49 Ceti was used as a reference star for PSF subtraction for the A- and B-type targets of the survey. The images were acquired with the NIC2 camera $\left(0{ }^{\prime \prime} 07565\right.$ pixel $\left.^{-1}\right)$ in the F110W filter, using the 0 !' 3 -radius coronagraphic mask. As it was selected as a reference star, 49 Ceti was observed at only one orientation of the spacecraft. The total exposure time is $2336 \mathrm{~s}$ (11 exposures).

The first SPHERE (Beuzit et al. 2008) data set was obtained on UT-2015-10-03 as part of the SHARDDS program, a search for circumstellar disks around nearby stars with large infrared excesses (VLT 096.C-0388(A) and 097.C-0394(A); PI: J. Milli). The images were acquired with the IRDIS instrument (Dohlen et al. 2008; 0."01225 pixel $^{-1}$ ) with an apodized Lyot coronagraph of radius 0".0925. The target was observed in pupil-stabilized mode, using the $\mathrm{H}$ broadband filter. The total exposure time is $1920 \mathrm{~s}$ (480 exposures), and the field rotation through the observations was $53^{\circ}$.

Additional SPHERE data were acquired on UT-2015-10-04 in IRDIFS mode to search for giant planets within the disk (VLT 096.C-0414(A); PI: M. Booth). Exposures were obtained with IRDIS in dual-band mode (Vigan et al. 2010) with two narrowband methane filters $(\mathrm{H} 2: 1.593 \mu \mathrm{m}, \mathrm{H} 3: 1.667 \mu \mathrm{m})$, while low-resolution spectra were simultaneously obtained across the Y-J bands with the IFS (39 spectral channels between 0.95 and $1.38 \mu \mathrm{m}$; Claudi et al. 2008). The total sky rotation achieved in pupil-tracking mode was $105^{\circ} .4$ in 416 exposures (6656 s total exposure time).

Satellite spots were imprinted on the first and last images of each SPHERE sequence by its deformable mirror to locate the star and field rotation center. Unocculted stellar PSFs were acquired to calibrate the instrument photometric responses.

\section{Data Reduction}

The NICMOS data were processed as part of the Archival Legacy Investigations of Circumstellar Environments ALICE program (HST-AR-12652; PI: R. Soummer) and reduced with the ALICE pipeline (Choquet et al. 2014). We assembled a library of 740 images, gathering the F110W PSFs of all the reference stars contemporaneous to our data set in the NICMOS archive (58 stars). Using the $80 \%$ of this library most correlated with 49 Ceti data, we used the KLIP algorithm (Soummer et al. 2012) to subtract the star PSFs from the images, built out of the 136 first eigenmodes of the library $(23 \%)$. The area within a radius of 10 pixels from the star was excluded from the reduction. The final image results from the mean of the individual exposures weighted by their exposure times. The signal-to-noise ratio per resolution element (SNRE) is computed as described in Rodigas et al. (2012).

Each SPHERE data set was preprocessed with the SPHERE Data Reduction and Handling pipeline (Pavlov et al. 2008). To image and characterize the disk, the IRDIS H-band data were reduced by subtracting the mean of the data cube from each individual image (cADI; Marois et al. 2006). No exclusion angle was used to limit disk self-subtraction effects, as the processing throughput can be recovered with forward-modeling. The final image results from the mean-combination of all derotated reduced images. The SNRE map was also computed as in Rodigas et al. (2012). The noise estimations in both NICMOS and SPHERE data sets are thus directly comparable. The reduced images and the SNRE maps are shown in Figures 1(a) and (b).

To detect planet candidates, each SPHERE data set was filtered to remove circularly symmetric features like the stellar halo as described in Wahhaj et al. (2013). For speckle subtraction, the best 60 images matching the speckle pattern of each science image between 0 ". 2 and 0 ". 6 were selected as 
(a)

Reduced Images

(b)

SNRE maps

(c)

Best models

(d)

\section{Forward models}

(e)

Residual maps

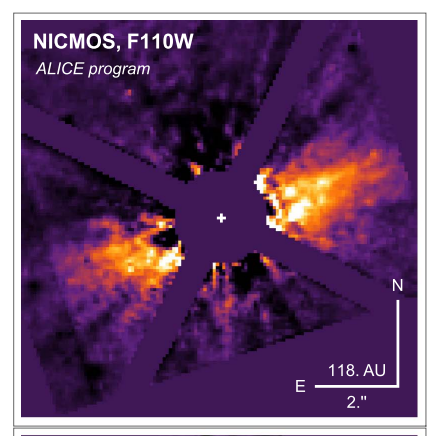

Surface Brightness $\left(\mu \mathrm{Jy} / \operatorname{arcsec}^{2}\right)$
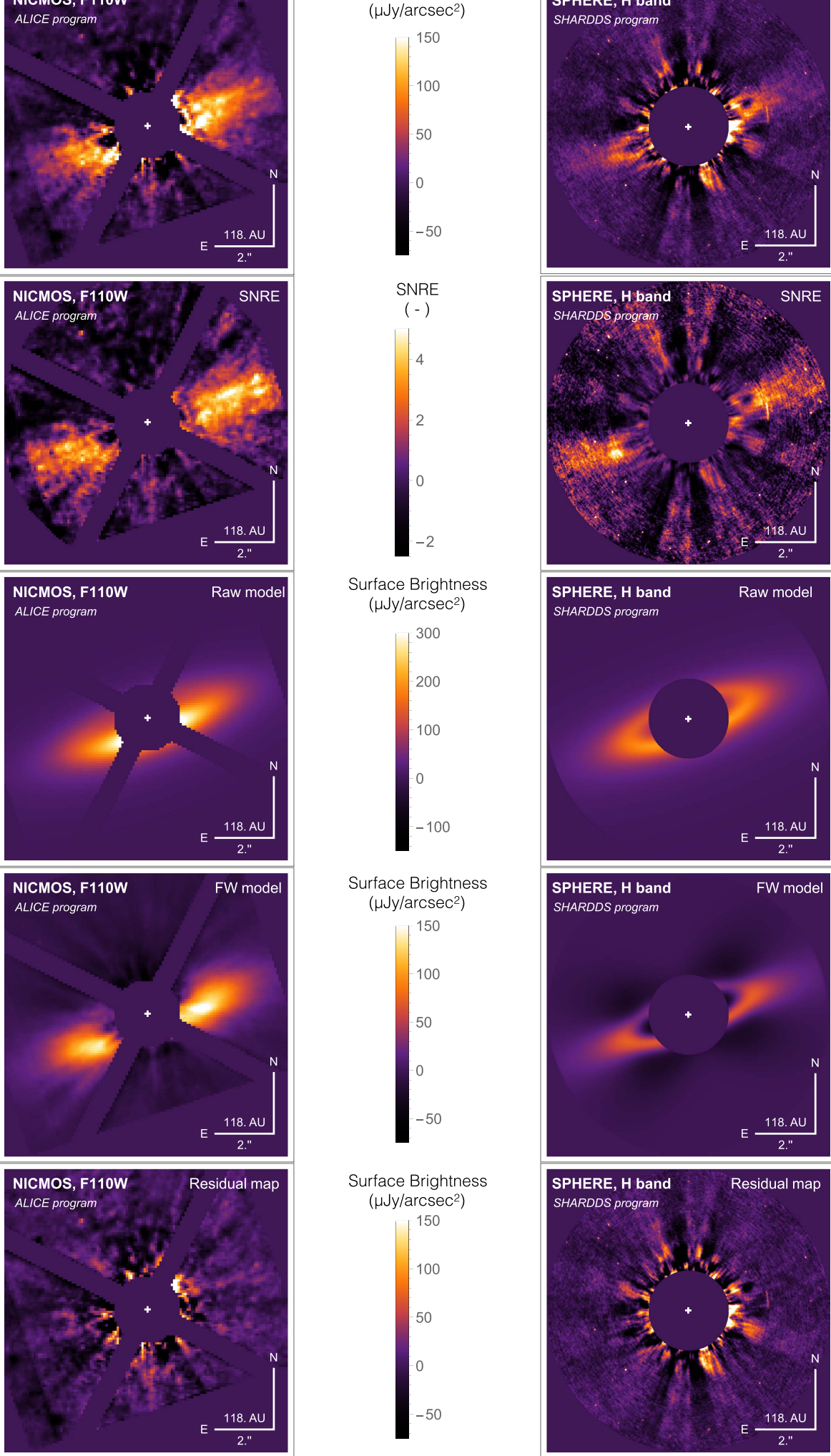

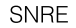

SNRE
$(-)$

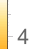

$-4$
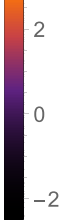

Surface Brightness $\left(\mu \mathrm{Jy} / \operatorname{arcsec}^{2}\right)$

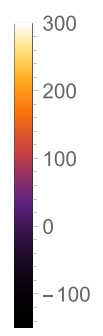

Surface Brightness $\left(\mu \mathrm{Jy} / \operatorname{arcsec}^{2}\right)$

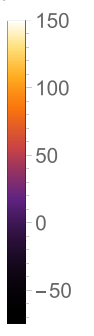

Surface Brightness $\left(\mu \mathrm{Jy} / \operatorname{arcsec}^{2}\right)$

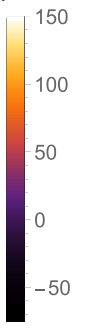

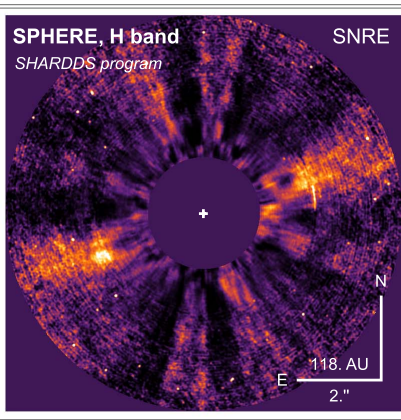
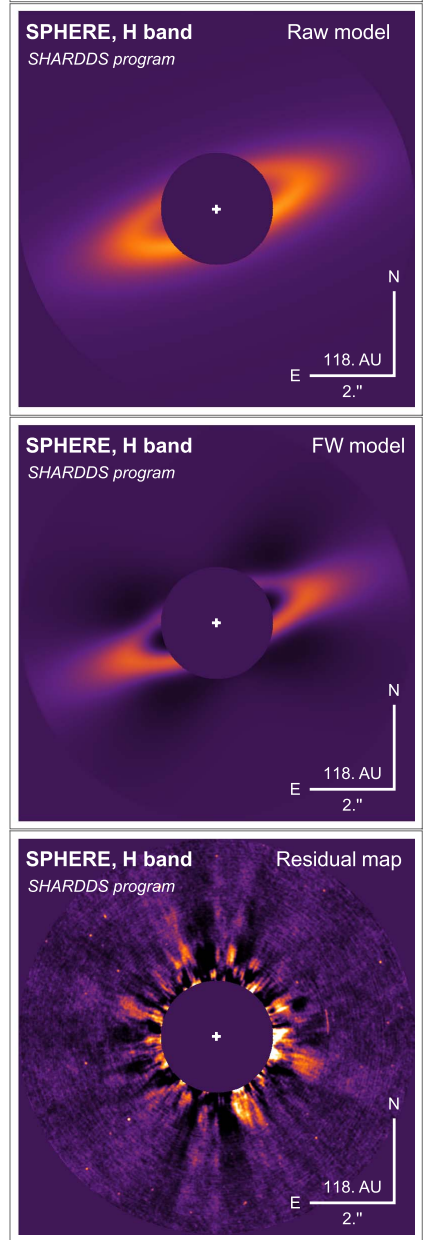

Figure 1. Scattered-light images of 49 Ceti's outer disk obtained with HST-NICMOS in the F110W filter and VLT-SPHERE in H band. (a) Reduced images of the disk, smoothed by convolution with an unocculted PSF. (b) Signal-to-noise ratio per resolution element maps. (c) Best models of the disk derived from forward modeling. (d) Best models after forward modeling, smoothed by convolution with a PSF. (e) Residual maps obtained by subtracting the best forward models (d) from the reduced images in (a).

reference images (in the speckle-aligned frames for the IFS). No more than $25 \%$ of them were allowed to overlap with the science image to within $1 \lambda / D$, to mitigate self-subtraction of the astrophysical signal (Wahhaj et al. 2016). The median of these reference libraries was subtracted from the corresponding science image, then all reduced images were derotated and 
median-combined. Only the $\mathrm{H} 2$ data were combined out of the $\mathrm{H} 2-\mathrm{H} 3$ data set, as the $\mathrm{H} 3$ frames only contribute to the noise for methane-rich planets (although the $\mathrm{H} 3$ frame were included in the reference libraries to improve speckle subtraction).

\section{Results}

49 Ceti's disk is detected at high confidence level in both data sets, with signal-to-noise ratios of 56 and 156 integrated over the whole disk, respectively, in the NICMOS and SPHERE images. The SPHERE image has an angular resolution 2.3 times better than the NICMOS one. The disk is detected between 1!"7 and 4!"6 (100-275 au) from the star, reaching $\mathrm{SNRE} \geqslant 5$ in some resolution elements. This corresponds to the same extent detected in thermal emission, albeit with a much finer angular resolution. The brightest parts of the disk (SNRE $\geqslant 4$ ) directly overlap with the CO emission resolved by Hughes et al. (2008). The disk is seen at an inclination significantly different than edge-on, consistent with the estimation by Moór et al. (2015b). We do not detect the inner disk, located at separations dominated by starlight residuals behind the reduction masks $\left(\leqslant 1^{\prime \prime}\right)$.

No point source is detected in the data sets. Fake sources were injected in the SPHERE raw data to estimate our detection limits (Figure 2). The sources had contrasts uniform across Y-J (worst-case scenario) and were 10 times brighter in $\mathrm{H} 2$ than in $\mathrm{H} 3$ to simulate methane-rich planets (best-case scenario). Using AMES-Cond evolutionary models (Baraffe et al. 2003), we rule out companions more massive than $3 M_{\text {Jup }}$ beyond 20 au (within and beyond the inner disk) and than $1 M_{\text {Jup }}$ beyond 110 au (within the outer disk).

\section{Analysis \\ 5.1. Disk Modeling}

We analyzed our images with a model grid fitting procedure. Our objectives are to: (1) constrain the main morphological characteristics of the disk; (2) investigate the significance of a possible east-west brightness asymmetry and of a continuous versus ring-like radial profile with respect to PSF-subtraction artifacts; (3) quantify the disk surface brightness (SB) and color. We modeled the NICMOS and the SPHERE images independently, using simple morphological disk models. The dust properties will be analyzed in a subsequent publication.

We used the GRaTer radiative transfer code (Augereau et al. 1999; Lebreton et al. 2012) to create scattered-light images of optically thin centro-symmetric disks assuming Henyey \& Greenstein (1941, hereafter HG) anisotropic scattering. We assumed a Gaussian profile for the vertical dust density distribution with a constant aspect ratio $h=0.05$ (expected for unperturbed debris disks; Thébault 2009), and we varied six parameters to fit the NICMOS image: the parent belt radius $R_{0}$, the inclination $i$, position angle (PA) $\theta$, the HG factor of scattering asymmetry $|g|$, and the radial density distribution power laws $\alpha_{\text {in }}$ and $\alpha_{\text {out }}$, respectively, inward and outward from the parent radius. We varied the same parameters to fit the SPHERE image, except the PA that we fixed to $110^{\circ}$, the best value found for the NICMOS image, to decrease the computation time. The boundaries and sampling of each grid are described in Table 1.

To calibrate the PSF-subtraction throughput and artifacts, we used the forward-modeling methods described in Choquet et al. (2016) and Milli et al. (2012) for the NICMOS and SPHERE
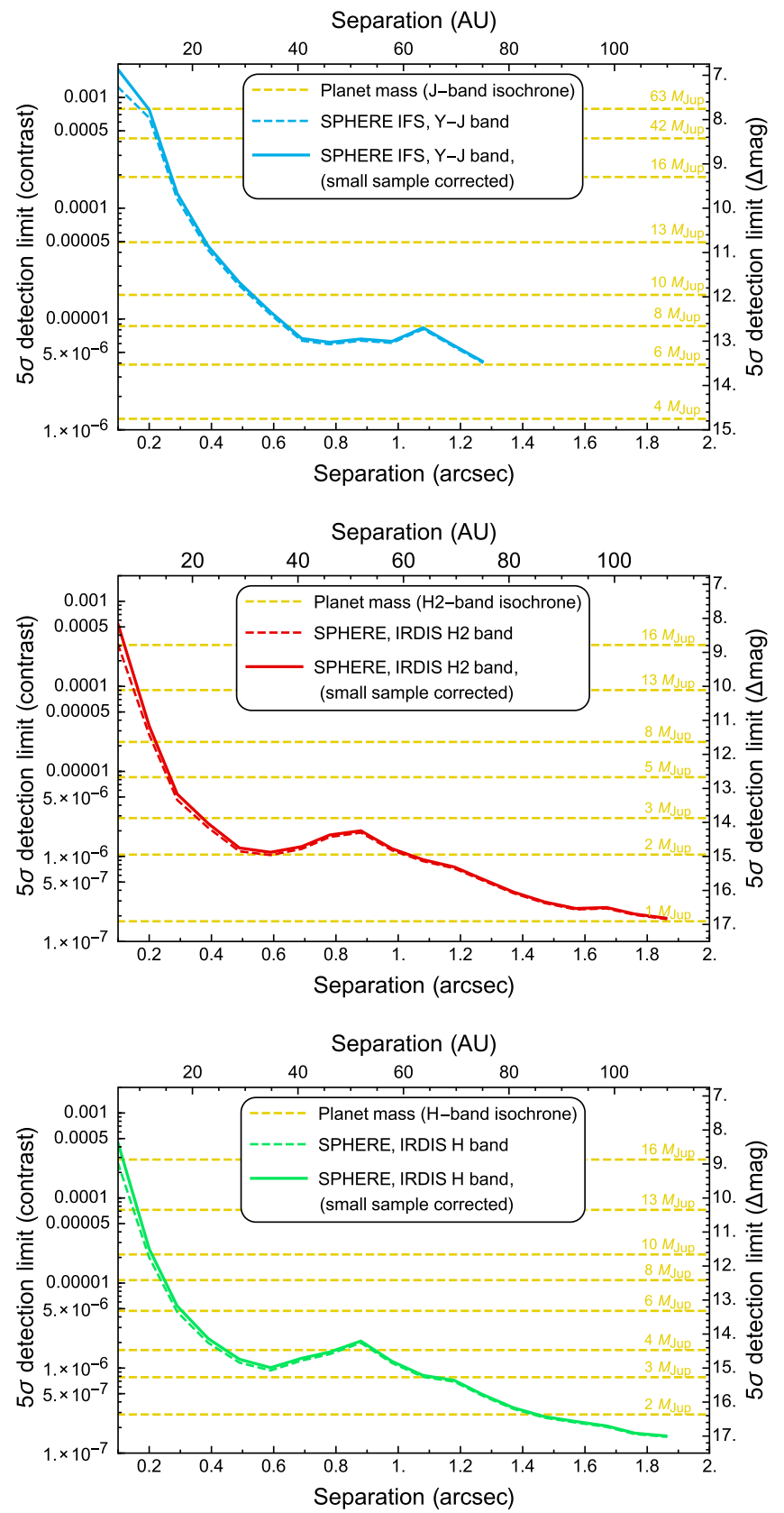

Figure 2. Point-source detection limits around 49 Ceti inferred from the SPHERE data corrected from small-sample statistics (Mawet et al. 2014). Planet-mass conversions are from AMES-Cond evolutionary models (Baraffe et al. 2003).

images, respectively. The forward-model intensities were scaled to the same fluxes as the images within ellipses slightly larger than and including the disk, and reduced chi-square values $\chi_{\text {red }}^{2}$ were computed within the same area: 1 "! $8 \times 5$ ". 2 semiminor and semimajor axes in the NICMOS image, and 1 !" $4 \times 5$ ". 4 in the SPHERE image. Circular areas of radii 1" 1 and 1."4 from the star, respectively, were excluded. The corresponding numbers of degrees of freedom $\left(N_{\text {dof }}\right)$ are respectively 3571 and 103,790. The best models, forward models, and residual maps are shown in Figures 1(c)-(e). The parameters of the best models of the grids are reported in Table 1, along with refined values and uncertainties computed by interpolating the $\chi_{\text {red }}^{2}$ values around the best model. The 
Table 1

49 Ceti's Disk Modeling

\begin{tabular}{|c|c|c|c|c|c|}
\hline Param. & Min. & Max. & $N_{\text {val }}$ & Best Model & $\begin{array}{l}\text { Best Model } \\
\text { (interpolated) }\end{array}$ \\
\hline \multicolumn{6}{|c|}{ NICMOS image modeling: 7680 models, $N_{\text {dof }}=3571$} \\
\hline$R_{0}(\mathrm{au})$ & 150 & 190 & 5 & 170 & $166_{-15}^{+17}$ \\
\hline$i\left(^{\circ}\right)$ & 68 & 78 & 6 & 74 & $74.5_{-3.2}^{+2.6}$ \\
\hline$\theta\left({ }^{\circ}\right)$ & 106 & 112 & 4 & 110 & $109 \pm 2$ \\
\hline$|g|$ & 0.1 & 0.4 & 4 & 0.3 & $0.27 \pm 0.10$ \\
\hline$\alpha_{\text {in }}$ & 0 & 3 & 4 & 1 & $1.0_{-0.9}^{+0.7}$ \\
\hline$\alpha_{\text {out }}$ & -5 & -2 & 4 & -3 & $-3.2_{-0.8}^{+0.7}$ \\
\hline$\chi_{\text {red }}^{2}$ & $\cdots$ & $\cdots$ & $\cdots$ & 0.923 & $\cdots$ \\
\hline \multicolumn{6}{|c|}{ SPHERE image modeling: 4032 models, $N_{\text {dof }}=103790$} \\
\hline$R_{0}(\mathrm{au})$ & 120 & 180 & 7 & 130 & $129_{-9}^{+10}$ \\
\hline$i\left(^{\circ}\right)$ & 70 & 80 & 6 & 72 & $73 \pm 3$ \\
\hline$\theta\left(^{\circ}\right)$ & 110 & 110 & 1 & 110 & $\ldots$ \\
\hline$|g|$ & 0.0 & 0.3 & 4 & 0.1 & $0.11 \pm 0.06$ \\
\hline$\alpha_{\text {in }}$ & 1 & 6 & 6 & 3 & $2.6_{-1.3}^{+2.1}$ \\
\hline$\alpha_{\text {out }}$ & -4 & -1 & 4 & -2 & $-2.1 \pm 0.5$ \\
\hline$\chi_{\text {red }}^{2}$ & $\ldots$ & $\ldots$ & $\cdots$ & 0.966 & $\ldots$ \\
\hline
\end{tabular}

uncertainties correspond to the $1 \sigma$ deviation expected for a chisquare distribution $\left(\sqrt{2 N_{\text {dof }}}\right)$.

The fits are globally consistent with each other, although the SPHERE image fit presents a smaller disk radius $\left(129_{-9}^{+10}\right)$ than the NICMOS image fit $\left(166_{-15}^{+17}\right)$. This discrepancy may come from degeneracies with the surface density power-law parameters, as our uncertainties do not account for correlations between parameters. Both values are globally consistent with the dust thermal emission and $\mathrm{CO}$ gas emission extents. The SPHERE image fit prefers more isotropic scattering $(|g|=0.11 \pm 0.06)$ than the NICMOS fit $(|g|=0.27 \pm 0.10)$. This difference may be related to the dust properties. Both disk images are well fit by axisymmetric disk models, which demonstrates that the east/west brightness asymmetry observed in the NICMOS image is an oversubtraction artifact. Our best models show that the disk is inclined $73 \pm 3^{\circ}$ from face-on with a PA of $109 \pm 2^{\circ}$. These values are consistent with published geometries of both the inner and outer dust belts, but significantly differ from the $90 \pm 5^{\circ}$ inclination found for the $\mathrm{CO}$ gas emission by Hughes et al. (2008). The NICMOS image fit favors a shallow inward slope for the disk $\left(\alpha_{\text {in }}=1.0_{-0.9}^{+0.7}\right)$. The best SPHERE image fit has a consistent value, although more poorly constrained due to ADI post-processing artifacts. It is thus still unclear whether the gap seen in the SPHERE image is real or not.

\subsection{Disk Photometry}

Using the best model images (Figure 1(c)), we can estimate the disk photometry without being affected by PSF-subtraction biases. We measured disk integrated fluxes $F_{\text {scat }}$ of $2.5 \pm 0.9 \mathrm{mJy}$ in the NICMOS-F110W filter and $2.0 \pm$ $0.7 \mathrm{mJy}$ in the SPHERE H-band filter. Given the star luminosity in these bandpasses (11.9 Jy and $6.4 \mathrm{Jy}$, respectively), we estimate that the disk has a scattering efficiency of $F_{\text {scat }} / F_{\text {star }} \sim(2.1 \pm 0.7) \times 10^{-4}$ in F110W and $\sim(3.2 \pm 1.2)$ $\times 10^{-4}$ in $\mathrm{H}$ band. As the outer disk fractional infrared

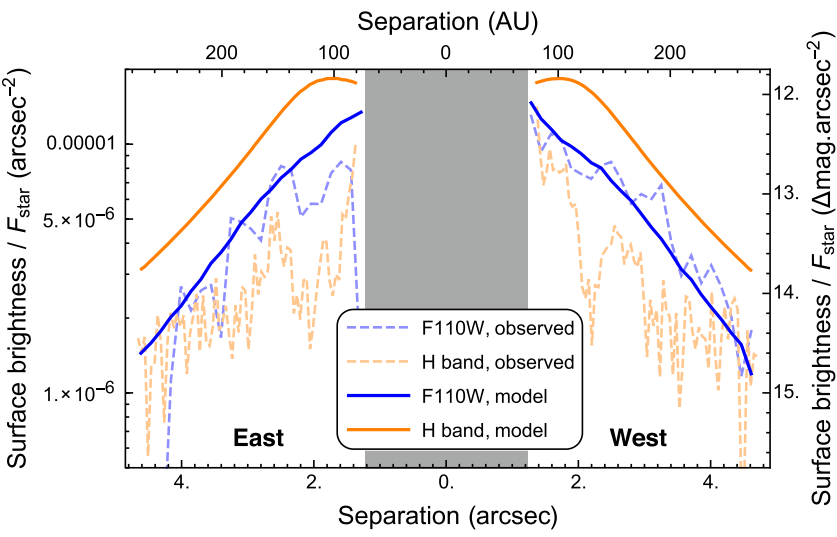

Figure 3. Disk reflectance radial profiles of 49 Ceti averaging forward and backward scattering, in NICMOS-F110W filter (blue) and SPHERE H-band filter (orange). The dashed and solid lines show the reflectance measured in the reduced images and in the best models, respectively. The increasing inward profile comes from the anisotropic scattering and morphology of the disk

luminosity is estimated to $L_{\mathrm{IR}} / L_{\mathrm{star}} \sim 9.0 \times 10^{-4}$ (Moór et al. 2015b), we computed a ratio $f_{\text {scat/IR }}$ of the integrated scattering efficiency over the integrated infrared excess of the disk of $0.2-0.4$. This ratio provides a degenerate combination of the dust albedo and phase function and is independent of the disk optical depth. Compared to other disks $\left(f_{\text {scat } / \text { IR }} \sim 0.75\right.$ on average in the visible; Schneider et al. 2014, Figure 8), 49 Ceti has a relatively low value that may point to dust with a low albedo and/or isotropic scattering.

We also estimated the SB of the disk in the two best models. In its brightest areas (within a factor of 5 of the peak values, $7.1 \operatorname{arcsec}^{2}$ and $14.2 \operatorname{arcsec}^{2}$, respectively), the disk's average $\mathrm{SB}$ is $129 \pm 14 \mu \mathrm{Jy} \operatorname{arcsec}^{-2}$ in F110W and $100 \pm 35 \mu \mathrm{Jy} \operatorname{arcsec}^{-2}$ in $\mathrm{H}$ band. In Figure 3, we present the disk SB radial profile normalized by the stellar flux. The SB profile was computed in rectangular apertures of size $1 \lambda /$ $D \times 2$ !" 3 along the disk's major axis, averaging the forward and backward scattering along the minor axis. As for the average SB and the integrated flux, the radial profiles show a slightly red color for the dust, consistent with a gray color.

\section{Discussion}

\subsection{Similarities with HD 131835}

49 Ceti's system shows interesting similarities with HD 131835, a 16 Myr A2IV subgiant star (de Zeeuw et al. 1999; Pecaut et al. 2012) also harboring a debris disk system. Both have high fractional infrared luminosities, well characterized by two dust populations at comparable blackbody temperatures and radii ( $\sim 40$ au for the inner disk and $\sim 150$ au for the outer disk), and with comparable dust masses $\sim 0.3 M_{\oplus}$ in the outer disk (Hung et al. 2015; Moór et al. 2015b). Both exhibit a significant amount of $\mathrm{CO}$ gas (Hughes et al. 2008; Moór et al. 2015a).

Our images of 49 Ceti now reveal that the outer disks in these two systems indeed extend over the same radial distance and are seen with the same $\sim 75^{\circ}$ inclination, which enables direct comparison of their scattering properties. Both disks seem to have similar anisotropy of scattering, especially when comparing our NICMOS 49 Ceti image with the GPI HD 131835 image, as none of them are biased by ADI artifacts. This may suggest that the two systems have similar dust compositions. However, HD 131835 was only detected in 

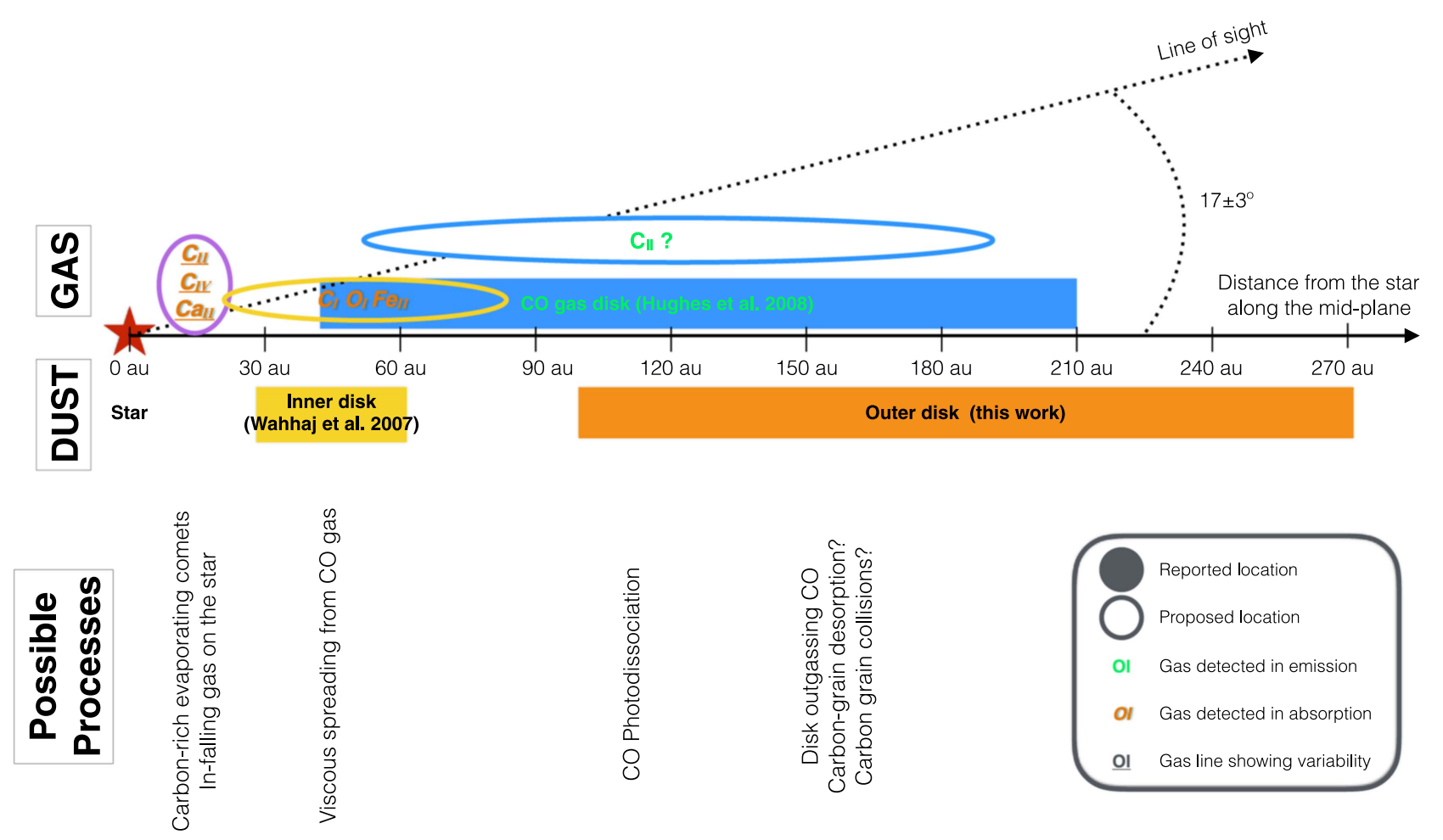

Figure 4. Schematic radial view of 49 Ceti's system through the mid-plane assuming an axisymmetric disk, gathering results from this work, previous studies, and thermodynamical model scenario. Atomic gas detections are from Montgomery \& Welsh (2012), Roberge et al. (2013, 2014), Malamut et al. (2014), and Miles et al. (2016).

polarized intensity, which may bias the comparison. Complementary images of both systems would be useful to confirm these similarities, as well as a combined study of 49 Ceti images and SED. We note that HD 131835's data are well fit by non-porous grains composed by a mixture of 1:1 carbon and silicate. Assuming both pure silicate and a 1:1 silicate-carbon mixture, we find with the GRaTer code that hard spheres larger than about $2 \mu \mathrm{m}$ have a gray scattering behavior similar to what is observed for 49 Ceti. This grain size is consistent with the $2.5 \mu \mathrm{m}$ blowout size expected for silicate grains around a $2 M_{\odot}$. star with luminosity $20.97 L_{\odot}$, and the $8 \mu \mathrm{m}$ blowout size of the silicate-carbon mixture. This indicates that gas drag is likely not affecting the grain dynamics even at the tail of the collisional cascade.

\subsection{Dust and Gas Location}

Our images of 49 Ceti at high angular resolution unambiguously show that the outer disk is not edge-on. This result has implications on the location of the stable atomic $(\mathrm{C}, \mathrm{O}, \mathrm{Fe})$ gas detected and $\mathrm{CO}$ gas not detected in absorption.

It indicates that the atomic gas may extend close to the star to intercept the line of sight (presumably within and/or interior to the inner disk) with a higher scale height than the dust. Conversely, as a significant amount of $\mathrm{CO}$ is detected in emission but not in absorption, it must be confined in the midplane at further radii, presumably in the outer disk. This is confirmed when comparing our images with the $\mathrm{CO}$ renzogram from Hughes et al. (2008). This atomic/molecular gas segregation is consistent with the scenario of second-generation gas production proposed by Kral et al. (2016), which can reproduce all gas observations in the $\beta$ Pic system (Matrà et al. 2017): because of very short photodissociation timescale, $\mathrm{CO}$ gas would only be located where it originates (outgassing comets in the outer disk), while atomic $\mathrm{C}$ and $\mathrm{O}$ gas, having much longer lifetimes, would spread inward and outward from viscous diffusion.

Our images do not clarify the location and origin of the C II gas emission. It may also result from CO-photodissociation, as well as from carbon-rich dust photodesorption or grain-grain collision as suggested by R13. Further analysis of 49 Ceti's dust composition would help investigate these questions.

We propose in Figure 4 a likely scenario for the gas and dust structure in 49 Ceti and physical mechanisms at work in the system. It summarizes previous findings as well as results from this work.

E.C. acknowledges support from NASA through Hubble Fellowship grant HST-HF2-51355 awarded by STScI, operated by AURA, Inc. under contract NAS5-26555, and support from HST-AR-12652, for research carried out at the Jet Propulsion Laboratory, California Institute of Technology. J.M. acknowledges ESO through the ESO fellowship program. M.B. acknowledges support from DFG project $\mathrm{Kr}$ 2164/15-1. G.M.K. is supported by the Royal Society as a Royal Society University Research Fellow. C.d.B. is supported by Mexican CONACyT research grant CB-2012-183007. L.M. acknowledges support by STFC through a graduate studentship. J.C.A. acknowledges support by the Programme National de Planétologie. We acknowledge support by the European Union through ERC grant 337569 for O.A. and C.A.G.G. and grant 279973 for M.W. and L.M.

\section{References}

Augereau, J. C., Lagrange, A. M., Mouillet, D., Papaloizou, J. C. B., \& Grorod, P. A. 1999, A\&A, 348, 557 
Baraffe, I., Chabrier, G., Barman, T. S., Allard, F., \& Hauschildt, P. H. 2003 , A\&A, 402, 701

Beuzit, J.-L., Feldt, M., Dohlen, K., et al. 2008, Proc. SPIE, 7014, 18

Chen, C. H., Mittal, T., Kuchner, M., et al. 2014, ApJS, 211, 25

Choquet, É., Perrin, M. D., Chen, C. H., et al. 2016, ApJL, 817, L2

Choquet, É., Pueyo, L., Hagan, J. B., et al. 2014, Proc. SPIE, 9143, 57

Claudi, R. U., Turatto, M., Gratton, R. G., et al. 2008, Proc. SPIE, 7014, 70143E

Dent, W. R. F., Wyatt, M. C., Roberge, A., et al. 2014, Sci, 343, 1490

de Zeeuw, P. T., Hoogerwerf, R., de Bruijne, J. H. J., Brown, A. G. A., \& Blaauw, A. 1999, AJ, 117, 354

Dohlen, K., Saisse, M., Origne, A., et al. 2008, Proc. SPIE, 7018, 701859

Greaves, J. S., Holland, W. S., Matthews, B. C., et al. 2016, MNRAS, 461, 3910

Henyey, L. G., \& Greenstein, J. L. 1941, ApJ, 93, 70

Hughes, A. M., Wilner, D. J., Kamp, I., \& Hogerheijde, M. R. 2008, ApJ, 681, 626

Hung, L.-W., Duchêne, G., Arriaga, P., et al. 2015, ApJL, 815, L14

Jura, M., Malkan, M., White, R., et al. 1998, ApJ, 505, 897

Kennedy, G. M., \& Wyatt, M. C. 2014, MNRAS, 444, 3164

Kral, Q., Wyatt, M., Carswell, R. F., et al. 2016, MNRAS, 461, 845

Lebreton, J., Augereau, J.-C., Thi, W.-F., et al. 2012, A\&A, 539, A17

Lieman-Sifry, J., Hughes, A. M., Carpenter, J. M., et al. 2016, ApJ, 828, 25

Malamut, C., Redfield, S., Linsky, J. L., Wood, B. E., \& Ayres, T. R. 2014, ApJ, 787, 75

Marino, S., Matrà, L., Stark, C., et al. 2016, MNRAS, 460, 2933

Marois, C., Lafrenière, D., Doyon, R., Macintosh, B., \& Nadeau, D. 2006, ApJ, 641, 556

Matrà, L., Dent, W. R. F., Wyatt, M. C., et al. 2017, MNRAS, 464, 1415

Mawet, D., Milli, J., Wahhaj, Z., et al. 2014, ApJ, 792, 97
Miles, B. E., Roberge, A., \& Welsh, B. 2016, ApJ, 824, 126

Milli, J., Mouillet, D., Lagrange, A.-M., et al. 2012, A\&A, 545, A111

Montgomery, S. L., \& Welsh, B. Y. 2012, PASP, 124, 1042

Moór, A., Ábrahám, P., Juhász, A., et al. 2011, ApJL, 740, L7

Moór, A., Henning, T., Juhász, A., et al. 2015a, ApJ, 814, 42

Moór, A., Kóspál, Á., Ábrahám, P., et al. 2015b, MNRAS, 447, 577

Pavlov, A., Feldt, M., \& Henning, T. 2008, in ASP Conf. Ser. 394, Astronomical Data Analysis Software and Systems XVII, ed. R. W. Argyle, P. S. Bunclark, \& J. R. Lewis (San Francisco, CA: ASP), 581

Pecaut, M. J., Mamajek, E. E., \& Bubar, E. J. 2012, ApJ, 746, 154

Roberge, A., Kamp, I., Montesinos, B., et al. 2013, ApJ, 771, 69

Roberge, A., Welsh, B. Y., Kamp, I., Weinberger, A. J., \& Grady, C. A. 2014, ApJL, 796, L11

Rodigas, T. J., Hinz, P. M., Leisenring, J., et al. 2012, ApJ, 752, 57

Sadakane, K., \& Nishida, M. 1986, PASP, 98, 685

Schneider, G., Grady, C. A., Hines, D. C., et al. 2014, AJ, 148, 59

Soummer, R., Perrin, M. D., Pueyo, L., et al. 2014, ApJL, 786, L23

Soummer, R., Pueyo, L., \& Larkin, J. 2012, ApJL, 755, L28

Thébault, P. 2009, A\&A, 505, 1269

van Leeuwen, F. 2007, A\&A, 474, 653

Vigan, A., Moutou, C., Langlois, M., et al. 2010, MNRAS, 407, 71

Wahhaj, Z., Koerner, D. W., \& Sargent, A. I. 2007, ApJ, 661, 368

Wahhaj, Z., Liu, M. C., Biller, B. A., et al. 2013, ApJ, 779, 80

Wahhaj, Z., Milli, J., Kennedy, G., et al. 2016, A\&A, 596, L4

Weinberger, A. J., Becklin, E. E., Schneider, G., et al. 1999, ApJL, 525, L53

Williams, J. P., \& Cieza, L. A. 2011, ARA\&A, 49, 67

Wyatt, M. C. 2008, ARA\&A, 46, 339

Zuckerman, B., Forveille, T., \& Kastner, J. H. 1995, Natur, 373, 494

Zuckerman, B., \& Song, I. 2012, ApJ, 758, 77 